\title{
Time measurement with the SND electromagnetic calorimeter
}

\author{
Vladimir Druzhinin ${ }^{1,2}$, Aleksandr Korol $^{1,2}$, Natalya Melnikova ${ }^{1,2, *}$, Sergey Serednyakov ${ }^{1,2}$, \\ and Ilya Surin ${ }^{1,2}$ \\ ${ }^{1}$ Budker Institute of Nuclear Physics, 11, Acad. Lavrentieva Pr., Novosibirsk, 630090 Russian Federa- \\ tion \\ ${ }^{2}$ Novosibirsk State University 1, Pirogova str., Novosibirsk, 630090, Russian Federation
}

\begin{abstract}
The SND is a non-magnetic detector deployed at the VEPP-2000 $e^{+} e^{-}$collider (BINP, Novosibirsk) for hadronic cross-section measurements in the center of mass energy range below $2 \mathrm{GeV}$. The important part of the detector is a three-layer hodoscopic electromagnetic calorimeter (EMC) based on $\mathrm{NaI}(\mathrm{Tl})$ counters. Until the recent EMC spectrometric channel upgrade, only the measurement of the energy deposition in counters was possible. New EMC signal digitizing electronics based on FADC allow us to obtain also the signal arrival time. The new electronics and supporting software, including digitized signal processing algorithms, are now used for data taking in the ongoing experiment. We discuss the amplitude and time extraction algorithms, the new system performance on experimental events and how this time measurement can be applied to physics analysis.
\end{abstract}

\section{Introduction}

The Spherical Neutral Detector (SND) [1-3] is one of the two detectors employed at the $e^{+} e^{-}$collider VEPP-2000 [4] in BINP (Novosibirsk, Russia). It was build for hadronic cross section measurement experiments and studies of hadron production dynamics, among others. The collider operates in the center of mass energy range from 0.3 to $2 \mathrm{GeV}$ using round beam optics. The maximum achieved luminosity after the collider upgrade [5] is $4 \times 10^{31} \mathrm{~cm}^{-2} \mathrm{~s}^{-1}$.

The SND is a general purpose non-magnetic detector, with a layout as shown in Fig. 1. It consists of a cylindrical tracking system, an electromagnetic calorimeter (EMC), threshold Cherenkov counters and a muon detector. The main part of the detector is the EMC. The calorimeter spectrometric channel has been upgraded recently, it's new electronics allows us to obtain a digitized signal shape which is processed with dedicated algorithms to extract energy and time information.

\section{The SND calorimeter}

The EMC consists of three layers of $1632 \mathrm{NaI}$ crystals and covers the polar angle range from $18^{\circ}$ to $162^{\circ}$ providing uniform particles detection in for $90 \%$ of the total solid angle (Fig. 2). Each EMC crystal has its own vacuum phototriod (VPT) attached to it for the detection of

\footnotetext{
*e-mail: N.A.Melnikova@inp.nsk.su
} 

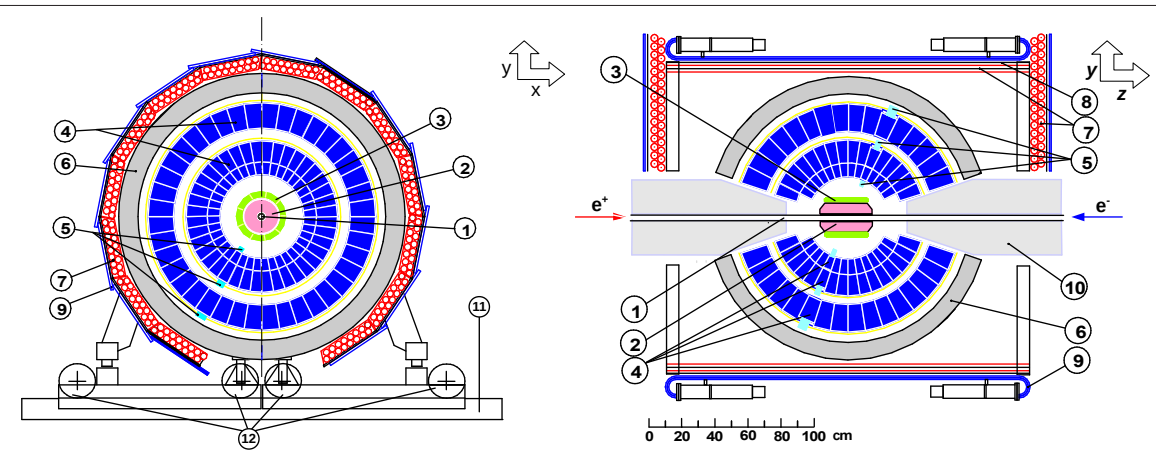

Figure 1. The SND scheme: 1 - vacuum pipe, 2 - tracking system (TS), 3 - threshold Cherenkov counter, 4-5 - electromagnetic calorimeter ( $\mathrm{NaI}(\mathrm{Tl}))$ (EMC), 6 - iron absorber, 7-9 - muon detector, 10 - focusing solenoids, 11 - rails, 12 - wheels.
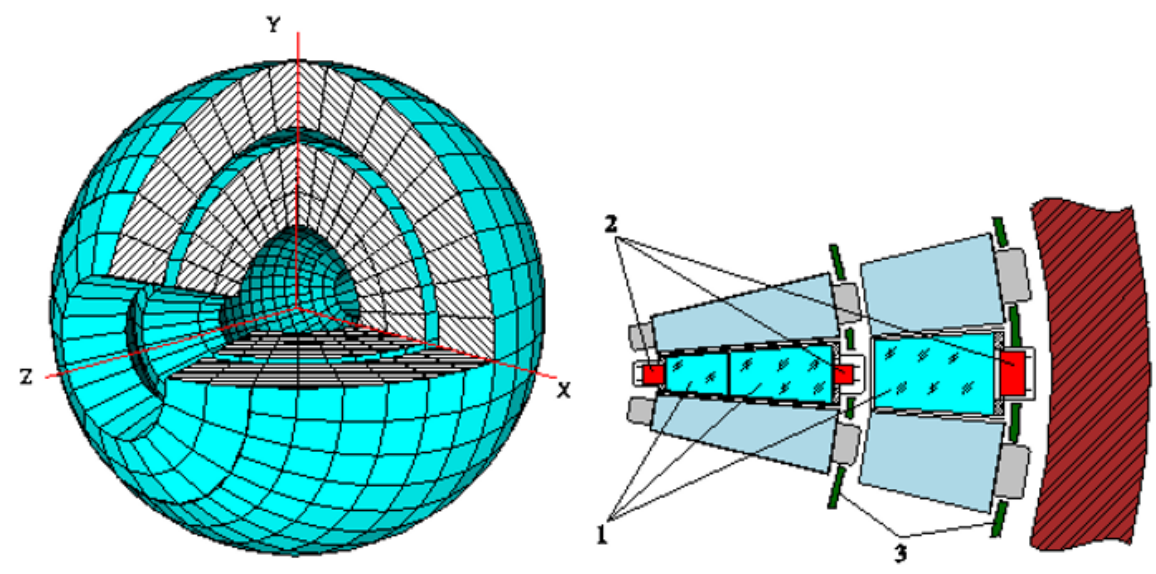

Figure 2. The 3D view of the SND EMC and the $\mathrm{NaI}(\mathrm{Tl})$ counters layout: 1 - vacuum $\mathrm{NaI}(\mathrm{Tl})$ crystals, 2 - vacuum phototriodes, 3 - aluminum supporting hemispheres.

scintillation signals (Fig. 2), together they form an EMC counter. All EMC counters form 160 EMC towers, where one EMC tower consists of up to 12 neighboring EMC counters from all three EMC layers.

\subsection{The EMC spectrometric channel}

The schematic layout of the new EMC spectrometric channel [6] is shown in Fig. 3. The electrical signals from the EMC counters come to charge-sensitive preamplifiers (CSA). The output signals are amplified and shaped inside F12 modules. Each F12 module processes signals from one EMC tower and provides an analog signal of the total energy deposition. Then these shaped signals are digitized by Flash-ADCs (FADCs) in our new Z24 modules. The Z24 module includes a Xilinx system on chip and six FADCs with four 12-bit channels each. The resulting digitized waveforms are read out after the arrival of a first-level trigger (FLT) signal. The FLT is synchronized with the beam revolution frequency $\left(f_{b r}=12.3\right.$ $\mathrm{MHz}$ ) and is formed using information from all F12 modules about energy deposition in 


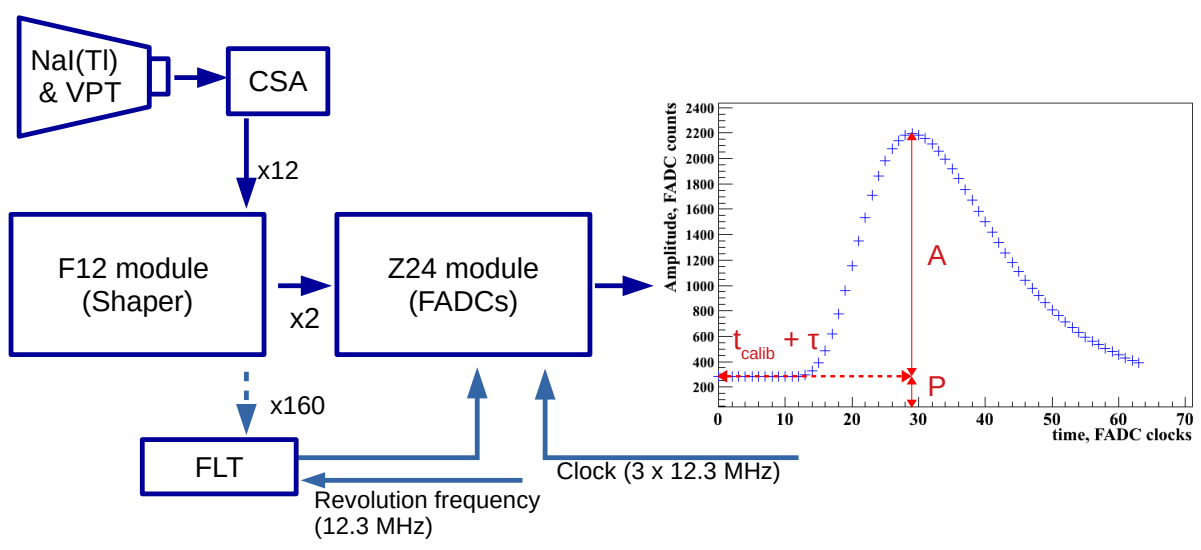

Figure 3. Schematic layout of the EMC spectrometric channel and a typical EMC signal shape. The abbreviations are described in the text.

EMC towers. The signal sampling is performed at a frequency of $3 \cdot f_{b r}$. These Z24 boards represent the main difference between the new and old EMC channels with ADCs measuring peak amplitudes. As a result, the waveform of the multiple sampled signal can be processed to extract its peak amplitude and time. The new EMC electronics have been in use for data taking in the experiments since the end of 2018.

\section{EMC signal processing}

A typical digitized EMC signal waveform ( Fig. 3 ) contains 64 samples with the sampling period $T_{s} \approx 27 \mathrm{~ns}$. A typical pedestal value is about 300 FADC counts with pedestal noise of $3 \div 5$ counts. One FADC count is roughly equal to $\sim 0.25 \mathrm{MeVs}$ (depending on the EMC channel).

F12 modules provide stable pulse shaping in all EMC channels, but the signal waveform may differ from one channel to another. A dedicated calibration procedure [7] is used to retrieve a normalized signal waveform without a pedestal and with a known peak location for each EMC channel. The shaped EMC signal can be well described with a function $U(t)$ :

$$
U(t)=A \cdot F(t-\tau)+P
$$

where $A$ - the signal amplitude, $F(t)$ - the function that describes the calibrated signal shape in the corresponding channel, $P$ - the signal pedestal and $\tau-$ signal arrival time with respect to the function $F$. The waveform is processed by fitting it with the function $U(t)$ to extract values of $A, P$ and the time shift $(\tau)$ between the calibrated signal shape and the real signal. At this moment, two different fitting algorithms are available. The first one is based on the linearization and is used for fast processing of most of the signals. The second one is an alternative algorithm under development. It is based on the correlation function and is expected to be able to handle special cases like saturated, heavily shifted and in other ways distorted signals. 

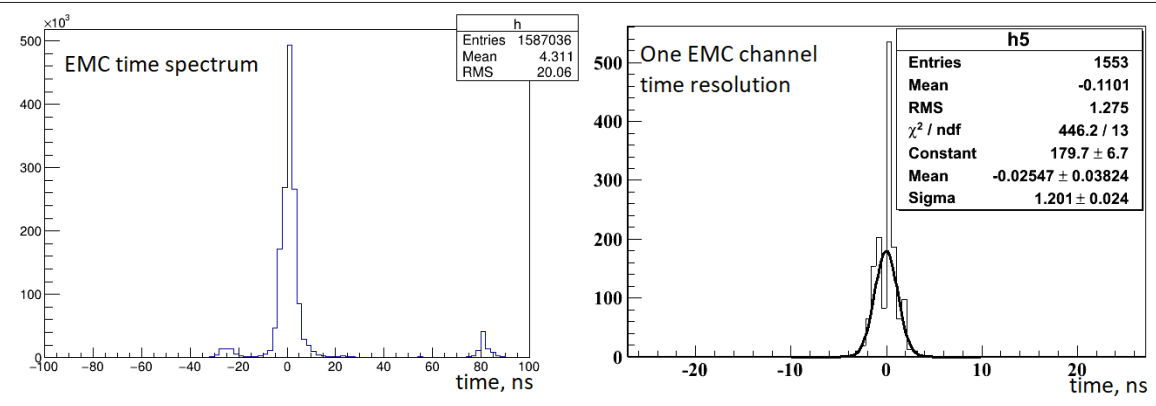

Figure 4. The linearization algorithm results on $e^{+} e^{-} \rightarrow e^{+} e^{-}$events. On the left - the signal times for all triggered EMC counters with the main peak around zero as expected, due to the signal shape calibration procedure being performed on the same process. On the right - the obtained time resolution in one EMC counter on fitted signals with reconstructed energy deposition $E>100 \mathrm{MeV}$.

\subsection{The linearization algorithm}

The linearization algorithm [8] is an adapted version of an algorithm developed for the Belle II calorimeter electronics [9]. It is optimized for using inside the Z24 module on FPGA. It minimizes the following $\chi^{2}$ function:

$$
\chi^{2}(t)=\left(y_{i}-A \cdot F\left(t_{i}-\tau\right)-P\right) \cdot S_{i j}^{-1} \cdot\left(y_{j}-A \cdot F\left(t_{j}-\tau\right)-P\right)
$$

where $y_{i}$ are signal samples with $i=[0,63], F\left(t_{i}\right)$ is the value of the calibrated signal shape function calculated at the $i$-sample time point $\left(t_{i}\right)$, and $S$ is a noise covariance matrix. The function $F(t)$ is linearized on a time grid with a time step of $\frac{1}{50} T_{s}$, allowing all coefficients needed for the minimization to be calculated in advance. This algorithm is relatively fast $(\sim 0.15 \mathrm{~ms}$ per signal) and successfully processes most of the strong enough signals $(\sim$ $8.5 \%)$, but the resulted time determination accuracy is limited by the time grid used for the linearization. For signals with $10<A<50$ FADC counts $(\sim 19 \%)$ only values for $A$ and $\tau$ are obtained with $P$ being fix to the calibrated pedestal value. The fit does not succeed for small signals with $A<10$ FADC counts $(\sim 71 \%)$. Signals with heavily shifted peaks, with FADC saturation $(\sim 0.004 \%)$ and other shape distortions also can't be processed. The result of this algorithm on data is shown in Fig. 4. There are several peaks that can be seen in the time spectrum obtained on $e^{+} e^{-} \rightarrow e^{+} e^{-}$events. The main peak is near zero because EMC channel signal shapes are calibrated using this type of events. The peak near $81 \mathrm{~ns}$ represents beam-induced background, which should be expected at $n \cdot 3 \cdot T_{s}(n \in \mathbb{Z})$ time points.

\subsection{The correlation function algorithm}

The correlation function algorithm processes a signal in two steps. First, it determines the time shift $(\tau)$ between a real signal and the calibrated signal shape in the corresponding EMC channel by searching for the maximum of the correlation function $(\omega(t))$ between them:

$$
\omega(\tau)=\sum_{i=0}^{i=63}\left(y_{i}-P\right) \cdot F\left(t_{i}-\tau\right) .
$$

In practice, we minimize $-\omega(\tau)$. For this minimization we utilize the GSL [10] implementation of the Brent's algorithm and GSL FFT methods for faster calculations. The main 

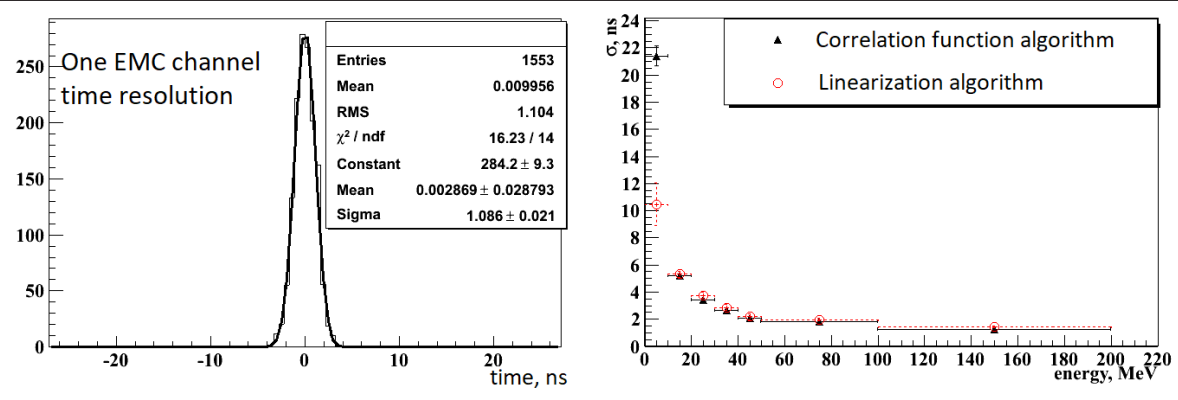

Figure 5. The correlation function algorithm results on $e^{+} e^{-} \rightarrow e^{+} e^{-}$events. On the left - the obtained time resolution in one EMC counter on fitted signals with reconstructed energy deposition $E>100$ $\mathrm{MeV}$ for the main time peak. On the right - time resolution dependence on EMC energy deposition for the same EMC counter obtained by two algorithms.

advantage of this algorithm lies in the improvement opportunities. At this moment, it is relatively slow ( $\sim 1.2 \mathrm{~ms}$ per signal), but it can successfully process almost all signals without any limitations on time accuracy. Although the algorithm has not been tested properly in realistic conditions.

The obtained time resolution for one EMC counter and its dependence on EMC energy deposition is almost the same as obtained with the linearization algorithm, the first results are shown in Fig. 5.

We plan to improve the algorithm to work correctly with saturated signals as these signals represent a significant part of the total energy deposition and should be processed with care. It is also important to process signals with long time delays that can't be processed by the linearization algorithm. It is our priority now to validate the algorithms on MC signals with known parameters using new simulation software that simulates EMC electronics response and can provide MC signal waveforms.

\section{Time measurement application}

The EMC time measurement provides the opportunity to identify interesting events while suppressing the rest using the time-of-light technique. The EMC time can be used for cosmicray background suppression due to the uniform time distribution of these events (no peak on the time spectra around zero). As it was mentioned in Sec. 3.1, beam-induced background can be suppressed as it mostly located at $n \cdot 3 \cdot T_{s}(n \in \mathbb{Z})$ time points (Fig. 4). Another important application is the identification of events with long EMC response times. As shown in Ref. [11], the EMC time can be used in physics analysis of $e^{+} e^{-} \rightarrow n \bar{n}$ events. Neutronantineutron pairs in the energy range of VEPP-2000 are non-relativistic so it could take about $8 \mathrm{~ns}$ for an antineutron with kinetic energy of $5 \mathrm{MeV}$ to annihilate in the first EMC layer. A first attempt was made to identify these events using EMC time on Monte Carlo simulation with the EMC electronics response. A good separation is seen between $e^{+} e^{-} \rightarrow n \bar{n}$ events and background events $\left(e^{+} e^{-} \rightarrow p \bar{p}, \gamma \gamma\right)$ in Fig. 6 .

\section{Summary}

The new EMC electronics provide digitized waveforms of the EMC signals that are processed by two dedicated algorithms to extract energy and time information: the linearization 

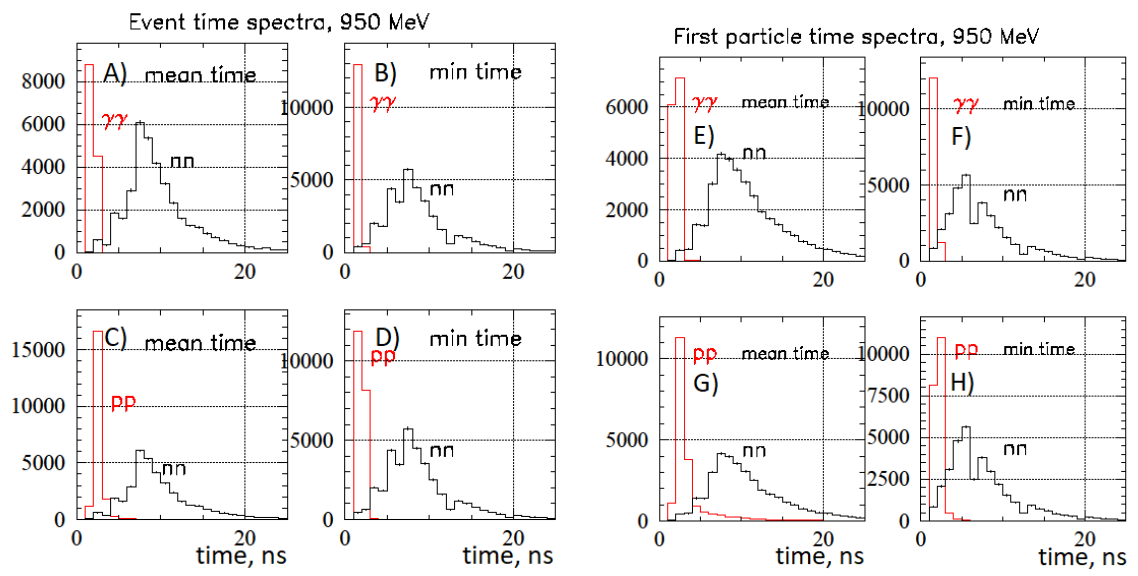

Figure 6. EMC time spectra for $e^{+} e^{-} \rightarrow n \bar{n}, e^{+} e^{-} \rightarrow p \bar{p}$ and $e^{+} e^{-} \rightarrow \gamma \gamma$ MC events with EMC signal shape simulation and fitting. The event time is a mean $(\mathrm{A}, \mathrm{C})$ or minimum $(\mathrm{B}, \mathrm{D})$ time of all successfully fitted EMC signals in the event. The first particle time is a mean $(E, G)$ or minimum $(F, H)$ time of all successfully fitted EMC signals that correspond to a particle with the highest energy deposition in the event.

algorithm and a new one based on the correlation function. The preliminary results of processing signals recorded during the 2018-2019 experimental season are presented for both algorithms. The achieved time resolution in the EMC counter is $\sim 1 \mathrm{~ns}$ for energy depositions of $100 \mathrm{MeV}$. The correlation function algorithm is still under development and will be validated on MC simulation of the EMC electronics response that has been implemented recently. It is expected that this algorithm will be able to handle signals that are difficult to process, such as signals with saturation. The possibility of $e^{+} e^{-} \rightarrow n \bar{n}$ event identification using EMC time has been demonstrated on MC data as an example of EMC time application in physics analysis.

\section{Acknowledgments}

The work is partially supported by the Russian Foundation for Basic Research (project nos. 18-02-00382-a, 18-02-00147-a and 20-02-00347).

\section{References}

[1] M.N. Achasov et al., Nucl. Instrum. Meth. A449, 125 (2000), hep-ex/9909015

[2] G.N. Abramov et al., eConf C010430, T10 (2001), hep-ex/0105093

[3] V.M. Aulchenko et al., Nucl. Instrum. Meth. A598, 102 (2009)

[4] B.I. Khazin (CMD-3, SND), Nucl. Instrum. Meth. A623, 353 (2010)

[5] Y. Shatunov et al., Physics of Particles and Nuclei Letters 15, 310 (2018)

[6] M. Achasov, et al., Nucl. Instrum. Meth. A824, 362 (2016), frontier Detectors for Frontier Physics: Proceedings of the 13th Pisa Meeting on Advanced Detectors

[7] M. Achasov et al., Nucl. Instrum. Meth. A936, 117 (2019), frontier Detectors for Frontier Physics: 14th Pisa Meeting on Advanced Detectors 
[8] I. Surin, V. Zhulanov, M. Achasov, V. Aulchenko, V. Druzhinin, V. Golubev, A. Korol, A. Bogdanchikov, S. Koshuba, D. Kovrizhin et al., Journal of Instrumentation 12, C07043 (2017)

[9] V. Aulchenko et al., Journal of Instrumentation 9, P09011 (2014)

[10] M. Galassi et al., Gnu scientific library reference manual (2018), https: //www .gnu. org/software/gsl/

[11] A. Bukin, D. Bukin, V. Golubev, S. Serednyakov, K. Skovpen, Y. Usov, Nuclear Instruments and Methods in Physics Research Section A: Accelerators, Spectrometers, Detectors and Associated Equipment 598, 264 (2009), instrumentation for Collding Beam Physics 